•生物编目・

\title{
中国植物采集先行者钟观光的采集考证
}

\author{
孟世勇 $1^{1 *}$ 刘慧圆 ${ }^{2,3}$ 余梦婷 4 刘全儒 ${ }^{3}$ 马金双 $5^{*}$ \\ 1 (北京大学生命科学学院, 北京 100871) \\ 2 (中国科学院植物研究所系统与进化植物学国家重点实验室, 北京 100093) \\ 3 (北京师范大学生命科学学院, 北京 100875) \\ 4 (北京大学哲学系, 北京 100871) \\ 5 (中国科学院上海辰山植物科学研究中心, 上海 201602)
}

\begin{abstract}
摘要: 基于文献与标本的研究, 本文重新梳理和考证了中国植物采集先行者钟观光先生的采集历史。钟观光是我 国第一个进行大规模植物标本采集的学者, 已知其最早的标本采自1912年(采集于北京), 最晚的是1933年(采集于 浙江)。目前能够确定的钟观光采集的馆藏标本计 10,228 份, 其中大部分保存于北京大学生物系植物标本室(PEY, 4,584份)、中国科学院植物研究所植物标本馆(PE, 2,966份)和北京师范大学生命科学学院植物标本室(BNU, 2,032 份), 共计 9,582 份, 约占钟观光馆藏标本总数的 $93.7 \%$ 。英国皇家植物园邱园植物标本馆、爱丁堡植物园标本馆、 美国哈佛大学植物标本馆等亦存有少量钟观光采集的标本(16份)。
\end{abstract}

关键词：植物采集; 钟观光; 植物标本; 标本馆

\section{Research on the plant collection of Kuan-Kuang Tsoong, the forerunner of Chinese plant collections}

\author{
Shiyong Meng ${ }^{1^{*}}$, Huiyuan Liu ${ }^{2,3}$, Mengting Yu ${ }^{4}$ Quanru Liu ${ }^{3}$, Jinshuang $\mathrm{Ma}^{5^{*}}$ \\ 1 School of Life Sciences, Peking University, Beijing 100871 \\ 2 Institute of Botany, Chinese Academy of Sciences, Beijing 100093 \\ 3 College of Life Sciences, Beijing Normal University, Beijing 100875 \\ 4 Department of Philosophy, Peking University, Beijing 100871 \\ 5 Shanghai Chenshan Botanical Garden; Shanghai Chenshan Plant Science Research Center, Chinese Academy of Sci- \\ ences, Shanghai 201602
}

\begin{abstract}
Through literature and specimen research, we studied the collection history of Kuan-Kuang Tsoong, the forerunner of Chinese plant collections. Results showed that Kuan-Kuang Tsoong was the first scholar who conducted large-scale plant specimen collections in China and confirmed that the earliest specimen collected by Kuan-Kuang Tsoong was collected in 1912 (from Beijing) and the last specimens were collected in 1933 (from Zhejiang). Currently, 10,228 specimens collected by Kuan-Kuang Tsoong have been found, including specimens founds at the Herbarium, Department of Biology, Peking University (PEY, 4,584 sheets), Institute of Botany, Chinese Academy of Sciences (PE, 2,966) and Herbarium, College of Life Science, Beijing Normal University (BNU, 2,032). These herbaria preserved most specimens $(9,582$ sheets, accounting for $93.7 \%$ of the total). Our study also found that a small number of specimens collected by Kuan-Kuang Tsoong (16) were preserved by the Royal Botanic Gardens, Kew (K), the Edinburgh Botanic Garden (E), and Harvard University Herbaria (A).
\end{abstract}

Key words: collection; Kuan-Kuang Tsoong; plant specimen; herbarium

植物标本是植物分类学、系统进化生物学和生 态学研究的基础凭证, 也是植物资源开发的重要资
料。早在1840年第一次鸦片战争爆发以前，西方人 就已经开始研究中国的植物, 方法之一就是采集植

收稿日期: 2017-12-10; 接受日期: 2018-01-18

基金项目: 国家植物标本资源共享平台(2005DKA21401)

* 共同通讯作者 Co-authors for correspondence. E-mail: jinshuangma@gmail.com; msy542702@pku.edu.cn 
物标本, 其中比较著名的采集者有英国的Ernest $\mathrm{H}$. Wilson、Robert Fortune、Henry F. Hance、Augustine Henry、George Forrest, 法国的Père Jean Marie Delavay以及奥地利的Heinrich Handel-Mazzetti等(王 印政, 2004)。早年国外学者采集的植物标本以及依 据这些标本产生的研究成果至今仍影响着中国植 物分类学领域的发展。直到20世纪初, 中国学者才 开始进行相关的生物学调查工作。钟观光就是我国 最早开展现代生物学调查研究的学者之一(方文培, 1932; 胡先骕, 1935; 附录1)。

早期的考察和标本采集工作促进了大批新种 和重要物种的发现, 如珙桐(Davidia involucrata)、 观光木 (Tsongiodendron odorun) 以及多种珍贵杜鹃 花 (Rhododendron spp.) 等, 为中国现代分类学的建 立和发展发挥了重要的作用。为了历史传承, 有必 要系统地整理早期植物采集者的采集工作。国外在 这方面已经给我们树立了典范, 许多著名的西方采 集者的采集史都已得到整理(Bretschneider, 1898; Cox, 1945)。作为中国最早的著名植物采集者, 钟观 光的采集历史也一直备受关注：如郝景盛(1929)、 蒋维乔(1941)、于一飞和陈锦正(1988)、陈锦正和钟 任建(2012)等在各自的文章中对其采集工作均有一 定的介绍; 朱宗元和梁存柱(2005)从北京大学植物 标本室的建立出发探讨了钟观光的采集工作; 余梦 婷 $^{\circledR}$ 更是从钟观光的标本和日记出发, 尽可能详尽 地还原了其植物采集过程。然而, 上述研究大部分 是基于日记和回忆等文字资料, 标本依据主要基于 手稿或回忆录, 缺乏对现存标本的考证。本文在重 新研读相关文献的基础上, 借助中国植物标本数字 化的最新进展, 全面核实了目前能够收集到的所有 钟观光采集的标本信息, 试图更加详尽地总结其标 本采集的状况。

\section{1 资料收集与统计}

\section{1 文献数据收集}

文献的收集主要利用中国知网数据库、大成古 文期刊数据库、百度学术、邱园数据库等, 以钟观 光、科学仪器馆、K. K. Tsoong 或 K. Tsoong、中国 植物采集史等为关键词进行检索, 并查阅了当时著

(1) 余梦婷 (2015) 钟观光的植物采集和植物学研究工作, 10-24 页. 硕士学位论文, 北京大学, 北京.
名植物分类学家如胡先骕、方文培、郝景盛等以及 与钟观光有关联的人员如蒋维乔、蔡元培等发表的 文章。

\section{2 标本数据收集与考证}

中国早期植物标本的采集记录相当不完备, 许 多标本并不能确定是否系钟观光所采集, 给考证工 作带来了很大的难度。因此, 标本数据的收集分成 两个部分。第一, 数字化标本信息的收集。即主要 查询国家标本资源共享平台(NSII, http://www.nsii. org.cn/2017/home.php)、中国数字植物标本馆(CVH, http://www.cvh.ac.cn/) 以及北京师范大学生命科学 学院植物标本室(BNU)、浙江大学植物标本馆 $(H Z U)$ 、福建师范大学生命科学学院植物标本室 (FNU)、湖南师范大学动植物标本馆(HNNU)和北京 大学生物系植物标本室(PEY)的大量数字化信息; 同时还查询了中国科学院昆明植物研究所标本馆 $(K U N) 、$ 英国爱丁堡植物园标本馆(E)、英国自然历 史博物馆 $(\mathrm{BM})$ 、英国皇家植物园邱园植物标本馆 $(\mathrm{K})$ 、美国哈佛大学植物标本馆 $(\mathrm{A})$ 等各馆的数字化 信息。第二, 考证相关标本馆或标本室的标本。根 据钟观光的采集旅行日记及其经历等绘制采集路 线图, 结合其笔迹, 将一些不确定的标本进行比对 核实。根据采集地点更正一些采集时间有误的标本, 将一些没有标明采集人，但是采集地点和采集时间 与钟观光行程一致的标本处理为由钟观光采集。通 过笔迹与采集时间, 还尝试将钟补勤采集的标本与 钟观光采集的标本加以区分。并订正了一些错误的 信息, 如钟观光的标本是采用民国纪年, 根据这一 原则, 又将一些可疑标本做了甄别, 确保所得数据 的可靠性。

\section{3 标本数据统计分析}

首先, 根据采集年份进行统计, 分析钟观光采 集的标本在时间上的变化, 确定最早采集时间和最 晚采集时间; 其次, 统计采集地点, 分析钟观光采 集的标本在地理上的分布情况; 第三, 统计钟观光 标本的馆藏情况, 明确这些标本的分布与保存现状; 第四, 利用国际植物名称索引(IPNI)数据库, 统计 以钟观光姓氏命名的植物种数。

\section{2 结果}

通过对众多标本馆的数据库进行检索后发现, 采集人标记为钟观光、钟光观、 K. K. TSOONG、 
K. K. CHUNG、TSOONG、K. K. TOONG或K. K. Tsoon, Tsoong, Kuang-kuan, Prof. K. K. Ts’ong, Prof. tsoong、K. K. TSOONG、K. K. Tsoong的标本共有 10,228份, 主要馆藏于中国(10,209份), 美国和英国 有少许。而在国内, 北京大学生物系植物标本室的 馆藏(4,584份)占全部标本的 $44.8 \%$, 其次是中国科 学院植物研究所植物标本馆(2,966份)和北京师范大 学生命科学学院植物标本室(2,032份), 分别占 $29 \%$ 和 $19.9 \%$, 其余的标本保存于中国科学院华南植物 园植物标本馆(200份)等处(表1)。在国外，英国皇家 植物园邱园和爱丁堡植物园标本馆各保存有 3 份, 英国自然历史博物馆保存有 1 份, 美国哈佛大学植 物标本馆有7份, 美国国家标本馆(US) 2份。

根据目前掌握的标本信息, 钟观光最早的标本 于1912年采自北京(图1), 最晚的于1933年采自浙江 (图2)。1918-1921年是其采集工作最集中的时期, 每 年采集量为500份以上。按照年采集量和采集时间 可将钟观光的植物标本采集工作分成3个阶段：零星 采集阶段(1903-1917年)、大规模采集阶段(1918-
1921年)和后期采集阶段(1922-1940年)。

零星采集阶段(1903-1917年): 目前, 这一阶段 的标本我们仅找到28份(详细信息见表2)。早期标本 在制作方法上可能有所欠缺, 信息记录不完备, 也 没有得到妥善保存, 导致标本丢失严重, 即使存在, 也很难确定为钟观光采集。

大规模采集阶段(1918-1921年): 1917年, 蔡元 培任北京大学校长, 特聘钟先生为地质学系教授, 不授课, 专门采集植物标本, 为创立北京大学生物 系和标本馆做准备。1918-1921年, 钟观光以北京大 学全国生物调查员的身份, 历时四载, 途经福建、 广东、广西、云南、湖北、安徽、四川、河南、浙 江、江西和河北11省, 采集了约15,000号标本。我 们确认此时间段的标本有 4,323份, 占其全部标本 的 $42.3 \%$ 。虽然由于信息不全, 一些标本无法确定具 体采集时间, 但仍然占了全部标本的将近一半。基 于已有的标本信息，我们绘制了1918-1921年间钟 观光采集标本的省份分布图(图3)。如图3所示, 钟观 光在广东省采集的标本最多, 有 1,294 份, 而标本数

\section{表 1 钟观光采集标本在各标本馆的分布情况}

Table 1 Distribution of specimens collected by Kuan-Kuang Tsoong in Herbarium around the world

\begin{tabular}{|c|c|}
\hline $\begin{array}{l}\text { 标本馆 } \\
\text { Herbarium }\end{array}$ & $\begin{array}{l}\text { 标本数目 } \\
\text { Number of specimens }\end{array}$ \\
\hline 哈佛大学植物标本馆 Harvard Museum of Natural History (A) & 7 \\
\hline $\begin{array}{l}\text { 北京师范大学生命科学学院植物标本室 } \\
\text { Herbarium, College of Life Science, Beijing Normal University (BNU) }\end{array}$ & 2,032 \\
\hline 英国自然历史博物馆 The Natural History Museum (BM) & 1 \\
\hline 爱丁堡植物园标本馆 Royal Botanic Garden Edinburgh (E) & 3 \\
\hline 复旦大学生命科学学院植物标本室 Herbarium, School of Life Sciences, Fudan University (FUS) & 14 \\
\hline 河北师范大学生命科学学院植物标本室 Herbarium, College of Life Science, Hebei Normal University (HBNU) & 17 \\
\hline $\begin{array}{l}\text { 中国科学院华南植物园标本馆 } \\
\text { Herbarium, South China Botanical Garden, Chinese Academy of Sciences (IBSC) }\end{array}$ & 200 \\
\hline 英国皇家植物园邱园植物标本馆 Royal Botanic Gardens (Kew) (K) & 3 \\
\hline 中国科学院昆明植物研究所标本馆 Herbarium, Kunming Institute of Botany, Chinese Academy of Sciences (KUN) & 9 \\
\hline 南京大学生命科学学院植物标本室 Herbarium, School of Life Sciences, Nanjing University (N) & 9 \\
\hline $\begin{array}{l}\text { 江苏省中国科学院植物研究所标本馆 Herbarium, Institute of Botany, Jiangsu Province and Chinese Academy of } \\
\text { Sciences (NAS) }\end{array}$ & 22 \\
\hline 南开大学生命科学学院植物标本室 Herbarium, College of Life Science, Nankai University (NKU) & 6 \\
\hline 中国科学院植物研究所植物标本馆 Herbarium, Institute of Botany, Chinese Academy of Sciences (PE) & 2,966 \\
\hline 北京大学生物系植物标本室 Herbarium, Department of Biology, Peking University (PEY) & 4,584 \\
\hline 中山大学生命科学学院植物标本室 Herbarium, School of Life Sciences, Sun Yat-sen University (SYS) & 177 \\
\hline 四川大学生物系植物标本室 Herbarium, College of Life Sciences, Sichuan University (SZ) & 26 \\
\hline 美国国家标本馆 United States National Herbarium, Smithsonian Institution (US) & 2 \\
\hline $\begin{array}{l}\text { 西北农林科技大学生命科学学院植物标本馆 Herbarium, College of Life Sciences, Northwest Agriculture and For- } \\
\text { estry University (WUK) }\end{array}$ & 149 \\
\hline 浙江自然博物馆植物标本室 Herbarium, Zhejiang Museum of Natural History (ZM) & 1 \\
\hline 总共 Total & 10,228 \\
\hline
\end{tabular}




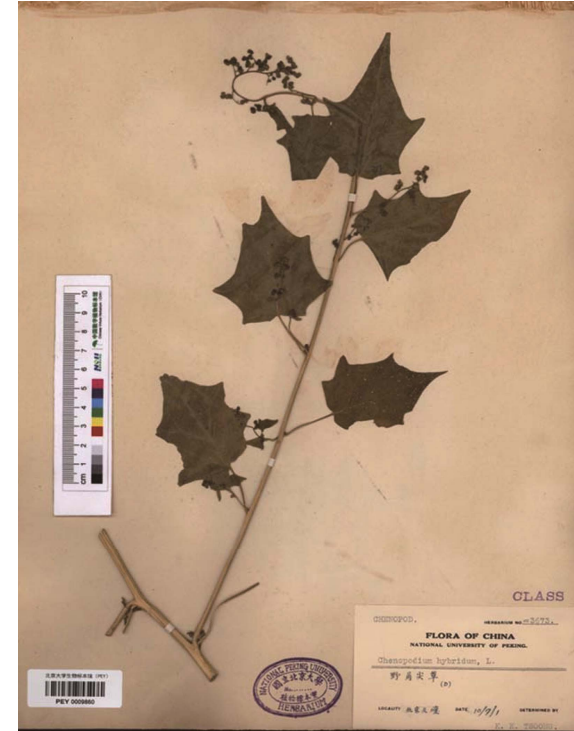

图 1 钟观光于 1912 年 7 月 10 日采自北京的标本(北京大 学生物系植物标本室)

Fig. 1 Specimen collected by Kuan-Kuang Tsoong in Beijing on July 101912 (Herbarium, Department of Biology, Peking University)

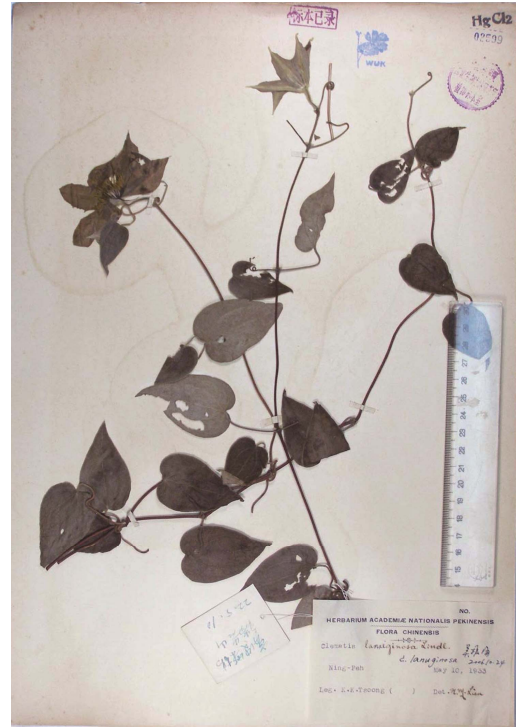

图 2 钟观光于 1933 年 5 月 10 日采自浙江的植物标本(西 北农林科技大学植物标本馆)

Fig. 2 Specimen collected by Kuan-Kuang Tsoong in Zhejiang on May 101933 (Herbarium, College of Life Sciences, Northwest Agriculture and Forestry University)

\section{表 2 钟观光在 1917 年及以前采集的标本信息}

Table 2 Information of specimens collected by Kuan-Kuang Tsoong before 1917

\begin{tabular}{llll}
\hline 年份 Year & 份数 No. & 采集地点(份数) Location (no.) & 现存储地点 Herbarium \\
\hline 1912 & 7 & 北京(3), 广东(3), 浙江(1) Beijing (3), Guangdong (3), Zhejiang (1) & 北京大学生物系植物标本室(7) PEY (7) \\
1913 & 4 & 北京(2), 浙江(1), 安徽(1) Beijing (2), Zhejiang (1), Anhui (1) & 北京大学生物系植物标本室(4) PEY (4) \\
1916 & 10 & 湖南(6), 浙江(4) Hunan (6), Zhejiang (4) & 北京大学生物系植物标本室(8), 中国科学院 \\
& 7 & 植物研究所标本馆(2) PEY (8), PE (2) \\
1917 & 7 & 浙江(5), 广东(2), 河南(1) Zhejiang (5), Guangdong (2), Henan (1) & $\begin{array}{l}\text { 北京大学生物系植物标本室(6), 中国科学院 } \\
\text { 华南植物园标本馆(1) PEY (6), IBSC (1) }\end{array}$ \\
\hline
\end{tabular}

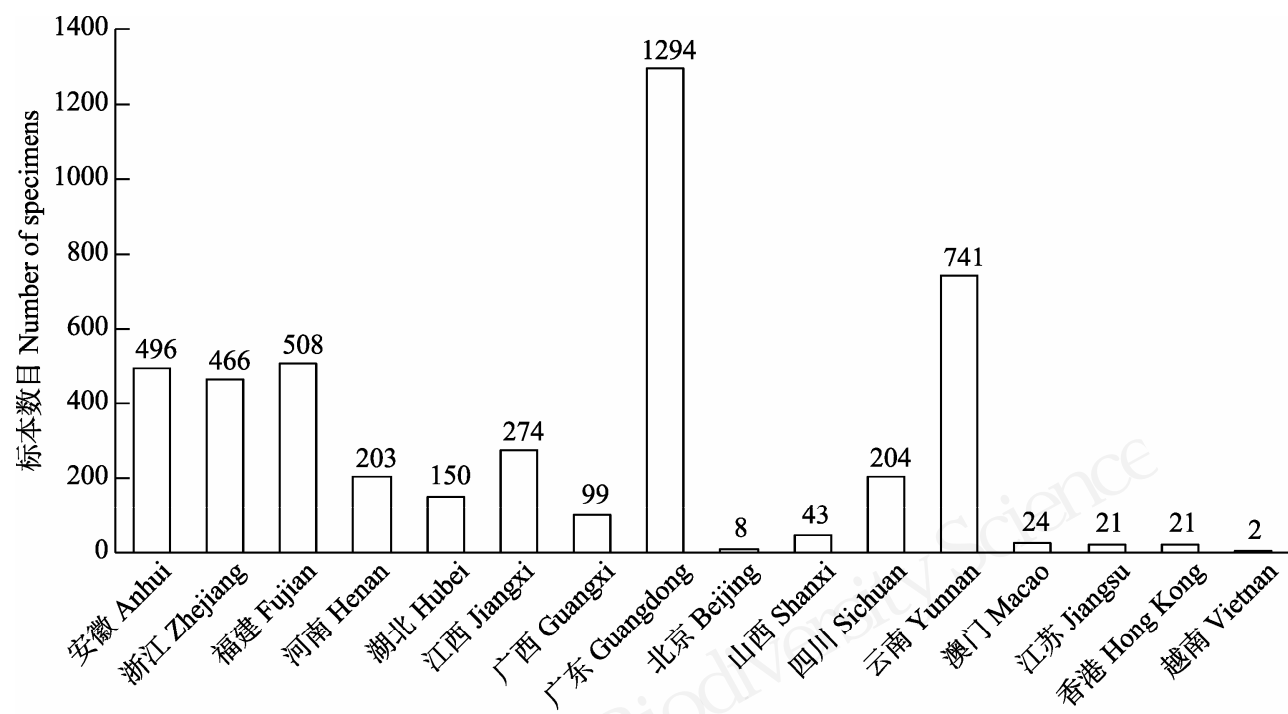

省份或国家 Province or country

图 3 1918-1921 年间钟观光标本采集地分布图

Fig. 3 Footprints of K. K. Tsoong from 1918 to 1921 
量在100份以上的省份有安徽、浙江、福建、河南、 湖北、江西、四川和云南。

后期采集阶段 (1922-1940 年): 钟观光于 1922-1940 年间采集的标本, 目前我们收集到了 354 份, 主要采集地点为浙江。其中采集时间最晚的是 1933 年 5 月 10 日采自其家乡浙江镇海柴桥的一份 毛叶铁线莲 (Clematis lanuginose) (标本条码号 WUK0003599, 图 2), 目前存放于西北农林科技大 学植物标本馆。

\section{3 结论}

\section{1 钟观光从哪一年开始采集植物标本?}

在中国的植物分类学发展史中, “钟观光既是 新植物学界(现代植物分类学) 的最初一人, 也是中 国旧植物学界(本草学)最后一人, 起到了承前启后 的作用, 中国植物的科名(中文名)有 $80 \%$ 以上由他 定名”(郝景盛, 1944)。然而, 关于钟观光从什么时候 开始采集植物标本这一重要问题, 却始终存在争 议。四川大学方文培(1932)认为“国人之采集植物, 当以北京大学钟观光氏和黄以仁氏为最早”, 但是 没有指明具体年份。著名植物分类学家胡先骕(1935) 认为“民国五年以后(即 1916 年), 则大规模之植物 采集与研究, 不得不推北京大学钟观光先生, 为得 风气之先”。钟先生的学生及好友蒋维乔(1941)回忆 “民元前七年(即 1904 年), 在科学仪器馆讲授理化, 而自赴杭州西湖闲居, 从此乃专心研究植物, 勤于 采集, 随时解剖”。在北平植物研究院与钟观光共事 过的植物分类学家郝景盛(1944)认为, 钟观光在光 绪末年(约 1908 年)即开始采集植物。曾任东吴大学 教授和台湾大学教授的著名植物分类学家李惠林 (Li Huilin)(1944)判断, 中国第一个系统地大规模采 集植物标本的植物学家应该是钟观光, 并认为钟观 光最早 1908 年就在广东及其他省份进行植物采集。 王宗训(1983)同样认可中国最早的标本采集学者为 钟观光, 并认为“他自 1910 年左右开始在湖南 高等师范学校和北大任教期间先后采集了约 15 万 号标本”。后来, 王宗训(1988)又明确提到“我国第一 位大规模采集标本的是钟观光, 他自 1913 年开始 采集, 历时十年.....”; 然而, 随着研究的深入, 王 宗训(1993)修正了自己的观点, 认为最早采集植物 标本的中国人可能是黄以仁(I-Jin Wang or I-Yen
Wong, 1874-1944) (1905 年采集); 朱宗元和梁存柱 (2005)根据钟观光曾在西湖养病及 Wang (1949)的 文章推测, 钟观光最早的采集年限在 1904-1905年间, 最迟不会晚于 1906 年。而最近, 余梦婷 ${ }^{\circledR}$ 根据钟观光 于 1901 年创办上海科学仪器馆, 且该馆在创办初 期就开始经营动植物标本推测, 钟观光接触植物标 本不晚于 1901 年(但不一定是自己亲自制作)。

本文第一作者于 2016年底在西北农林科技大 学植物标本馆(WUK)查看景天科标本时, 发现了一 份由上海科学仪器馆采集的植物标本(图4), 采集地 点是浙江天目山, 遗憾的是标本上没有载明具体采 集时间。随后作者在国家标本资源共享平台搜索到 内蒙古农业大学有6份由上海科学仪器馆采集的标 本, 成都中医药大学的1份洋常春藤 (Hedera helix) 标本则显示采集人为上海惠福里科学仪器馆标本 制作所。据谢振声(1989)考证, 以及曾就职于上海科 学仪器馆的张之铭(1947)回忆, 上海科学仪器馆创 办于1901年, 1903年设立制作所, 主要业务是仿制 和修理科学仪器，该馆又于1904年设立标本制作所, 专门制作动植物标本出售给各大学和中学用于教 学(谢振声, 1990)。以上提到的西北农林科技大学植

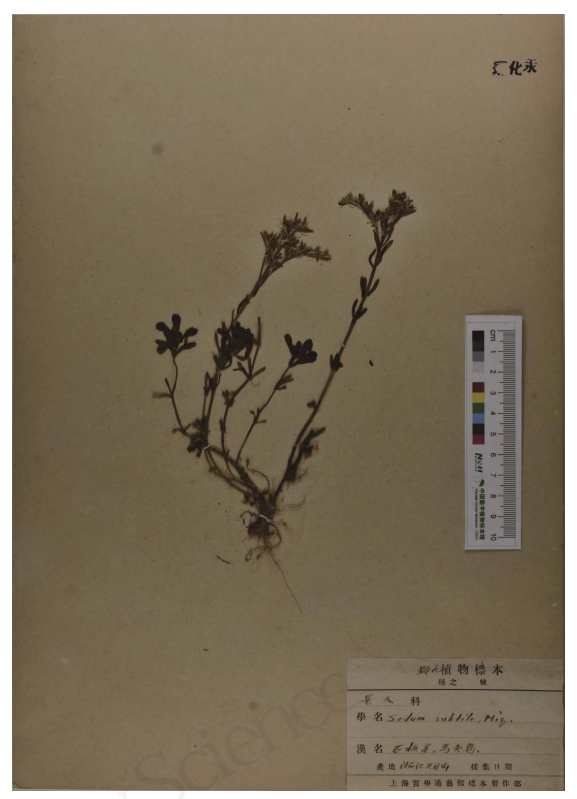

图 4 上海科学仪器馆采集的标本(西北农林科技大学植物 标本馆)

Fig. 4 Specimen collected by Shanghai Scientific Instruments Company (Herbarium, Northwestern Institute of Botany)

(1) 余梦婷 (2015) 钟观光的植物采集和植物学研究工作, 10-24 页. 硕士学位论文, 北京大学, 北京. 
物标本馆、内蒙古农业大学标本馆和成都中医药大 学标本馆所收藏的上海科学仪器馆采集的标本, 就 是这一时期采集制作的。1904年, 钟观光到西湖养 病, 自学李善兰编著的《植物学》, 开始对植物感 兴趣并进行研究(朱宗元和梁存柱, 2005)。据此我们 可以推测, 钟观光不晚于1904年就开始采集制作植 物标本。不无遗憾的是, 由于当时的标本制作不规 范, 上海科学仪器馆采集制作的这些标本基本信息 很不完善, 特别是没有记录采集人和具体采集时 间。所以, 暂且不能将这些标本归到钟观光名下, 只能待以后发掘更多资料再加以确定。

黄以仁是钟观光1901年在江苏南菁书院任教 时的学生(蒋维乔, 1941), 于1906-1909年留学日本 东京大学攻读植物学专业; 回国后于1918年参与编 写《植物学大辞典》, 后任职于河南大学(王宗训, 1993)。根据目前的资料, 黄以仁最早在1905年左右 开始采集植物标本并将其寄给日本学者鉴定(松田 定久, 1911)。我们在国家标本资源共享平台搜索到 第二军医大学标本馆有一份黄以仁在四川峨眉山 采集的台湾相思(Acacia confusa)标本(标本条码号 BH002081), 采集时间显示为1904年6月18日(图5)。 这也是目前最早由中国学者采集的有确切采集人 和采集日期的植物标本。黄以仁采集的标本主要保 存在日本(松田定久, 1911), 所以国内学术界对具体 情况不甚了解。此外, 京师大学堂在1905年左右也 设置了博物实习科, 请当时著名的植物学教授史部 氏授课(郝景盛, 1944)。据1905年的《录津报》记载, 京师大学堂师范馆设立标本制作处, 并招日本助手 堍田君来馆, 自制动植物标本。北京大学生物标本 馆的馆藏标本中, 就有一些由京师大学堂师范馆学 生采自北京百花山(图6)。以上这些是我们能够查到 的国人较早采集的标本, 但时间均晚于黄以仁的 1904年。

需要说明的是, 植物标本的采集不同于植物研 究。黄以仁(1906年以前)和钟观光早期(1908年以前) 的采集, 包括科学仪器馆等的采集, 严格说来还不 足以当作科学研究的依据。1912年, 钟先生受蔡元 培之邀, 入南京临时政府教育部任职, 后迁入北京, 其间节假日常同蔡元培、蒋维乔到南京和北京周边 山中采集植物(蒋维乔, 1941)。钟观光作为现代植物 分类学研究者去采集标本, 可以从1912年8月10日 采自北京的野角尖草(杂配藜Chenopodium hyb-
ridum)(标本条码号PEY0009860, 图1)算起, 这与蒋 维乔(1941)的回忆相吻合。这也可以说是中国植物

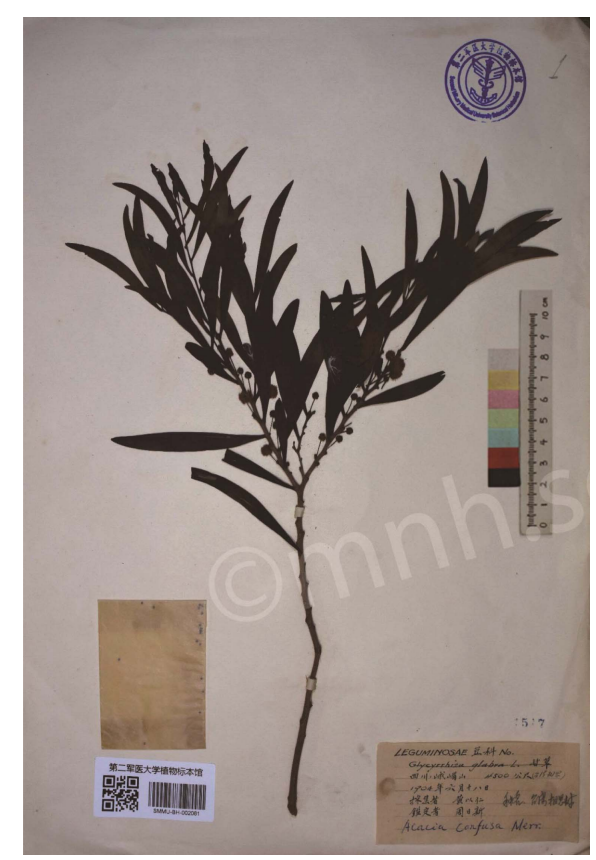

图 5 黄以仁采集的标本(1904 年 6 月 18 日, 第二军医大学 植物标本馆)

Fig. 5 Specimen collected by I-Jin Wang (June 18 1904, Herbarium, The Second Military Medical University)

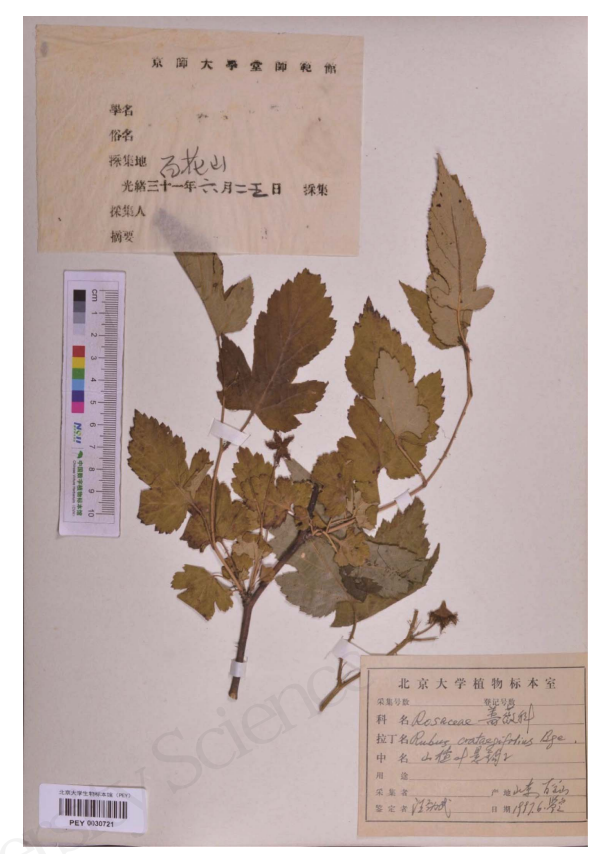

图 6 京师大学堂学生采集的植物标本(1905 年 6 月 25 日, 北京大学生物系植物标本室)

Fig. 6 Specimen collected by students of Imperial University of Peking (June 25 1905, Herbarium, Department of Biology, Peking University) 
分类学研究人员最早采集的标本了。Li (1944)认为 钟观光于1908年开始在广东采集标本, 然而我们并 没有找到相关的标本支持。据蒋维乔(1941)的回忆, 1908年前后, 钟观光正在上海开办理科实习学校, 培养小学教员。根据北京大学的标本采集记录, 钟 观光在民国7-8年在广东采集过标本(凭证标本条码 号: PEY0034334, 图7)。Li得出这一结论源于对钟观 光标本纪年方式的误解。钟观光采集的标本, 日期 标注方式主要有两种: 其一为“X/X/X”, 表示“日/月 /年”, 其二为“X.X.X”, 表示“年.月.日”, 所有日期均 采用民国纪年, 而非公元纪年 ${ }^{\circledR}$ 。如图7中, 24/8/7表 示民国7年8月24日采集, 即1918年8月24日。这一点 在研究中应予以注意。就钟观光的标本而言, 我们 发现各标本馆在标本信息数字化的过程中, 常常误 将民国年份直接记载为公元年份。1915年, 钟先生 受聘到湖南高等师范学校任博物学教授。他用近两 年的时间在湖南采集了约1,100号植物标本(蒋维乔, 1941; 朱宗元和梁存柱, 2005)。据此胡先骕推断其 最早采集植物标本的年份为1916年。王宗训(1983) 也是根据钟观光在湖南高等师范学校任教的时间 来推断其最早采集年限, 但他误将钟观光在湖南高

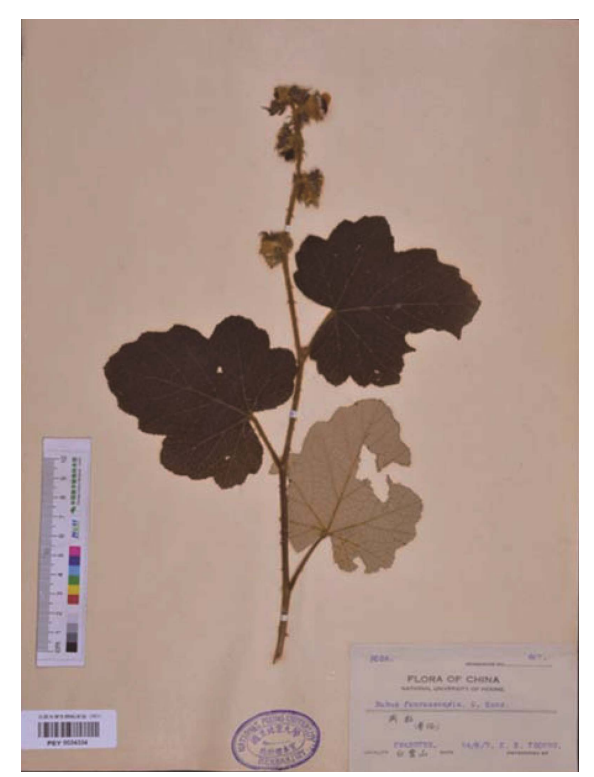

图 71918 年 8 月 24 日钟观光采自广东的标本(北京大学生 物系植物标本室)

Fig.7 Specimen collected by K. K. Tsoong in Guangdong at August 241918 (Herbarium, Department of Biology, Peking University)

(1) 余梦婷 (2015) 钟观光的植物采集和植物学研究工作, 10-24 页. 硕士学位论文, 北京大学, 北京.
等师范学校的工作时间判断为1910年或1913年。

\section{2 钟观光总共采集了多少份标本? 这些标本现 在何处?}

鉴于钟观光在我国植物标本采集史上的重要 性，详细了解其一生总共采集了多少份标本，目前 放在什么地方, 处于何种状态, 对于了解中国早期 植物学发展历史具有重要意义。静渊(1941)认为钟 观光一生采集的标本有 16,000 种, 而 Li (1944)认为 钟观光采集了 1,500 号标本, 结合目前已收集到的 钟观光标本, 1,500 显然不对。陈德㯟和曾令波 (1988)、王世珍等(2005)认为钟观光在 1917-1921 年间采集了 16,000 种、共计 150,000 号标本。任荣 荣(1980)、汪子春(1988)、范文涛和陈义产(1990)、 王宗训 (1983，1993) 均认为钟观光采集了超过 150,000 号标本, 并建立了北京大学植物标本室。朱 宗元和梁存柱(2005)认为钟观光采集了约 25,000 号, 其中北京大学 15,000 号, 浙江大学 7,000 号, 湖南 高等师范学校 1,100 号, 其他地方零星存有 1,200 号, 并指出前人观点可能都是依据汪发瓒先生 1949 年 在原北平研究院植物学研究所丛刊上的观点(Wang, 1949), 然而汪文可能由于印刷错误或笔误, 将 15,000 号误写为 150,000 号。陈锦正和钟任建(2012) 则认为钟观光一生采集标本共 30,000 号。遗憾的是, 以上判断均没有给出数据来源。北京大学生物系毕 业生郝景盛(1929)在北京大学 31 周年校庆时写道 “生物系植物标本整理出来的有八千多种”; 北京大 学生物系标本馆中有一份关于钟观光的介绍, 说他 历时四载, 途径皖浙粤桂等 11 省共采得 15,000 多 号腊叶标本。据余梦婷 ${ }^{1}$ 考证，钟观光在《北京大 学植物标本目录》中一共收录了 5,383 号标本, 还 有一些未经整理、未定名的标本。根据钟观光日记 中提到的单日最大采集量推算，他在大规模采集 阶段采集到的标本最多为 25,000 份。因此北京大 学标本馆的 15,000 份比较可信, 而 150,000 显然 是笔误。

综合以上内容, 我们不难发现, 在针对钟观光 的采集标本号数和物种数上存在两个不同的概念。 非分类学工作者经常将采集的号数、份数以及物种 数目相混淆, 其实这三者相差非常之大, 史学界、 传记作者或后人的回忆文章中，对这些概念的使用 尤其应该引起分类学工作者的注意。一号标本可能 
有一份或几份, 一号标本代表一个物种; 但是多号 标本不一定代表多个物种, 可能是一个物种也可能 是多个物种。澄清这些分类学的基本概念有助于避 免后续的考证工作似是而非甚至以讹传讹。

根据我们统计, 现有钟观光 10,228 份标本, 分 布中国、英国和美国 3 个国家, 保存于北京大学生 物系植物标本室等 19 家标本馆, 说明钟观光的标 本影响力大, 受到了普遍的重视。由于分布范围广, 也导致了标本搜索与考证的困难。在整理和考证钟 观光采集标本的过程中, 我们发现, 只有北京大学 生物标本馆的标本是钟观光亲自整理的, 并制作了 标本目录, 馆中标本的采集号和植物学名与《北京 大学植物标本目录》相对应。然而这份唯一记录钟 观光标本的目录上只有 5,383 号标本, 这与郝景盛
(1929)的回忆接近, 可见还有很大一部分标本未经 整理。这些没有整理的标本有一些被其他大学或研 究所收藏。据北京大学汪劲武教授回忆(汪先生和本 文第一作者个人交流), 钟观光的标本后来从北大 运走了一些。北京师范大学动植物标本馆里保存的 钟观光标本都是一些没有经过整理的标本, 因此 可以推断就是从北大运走的那些标本。在当时的条 件下, 这些运往他处的标本很可能没有得到妥善 整理和标记，而国内有些植物标本馆目前还没有实 现数字化, 因此这部分标本的信息我们尚且难以获 得。相信随着标本数字化工作的推进, 很可能有更 多钟观光采集的标本被整理出来。但是这里我们也 不得不承认, 这些标本也很可能因为种种原因已经 遗失。

\section{表 3 以纪念钟观光命名的植物名录}

Table 3 Checklist of plants in memory of Kuan-Kuang Tsoong

\begin{tabular}{|c|c|c|c|}
\hline $\begin{array}{l}\text { 序号 } \\
\text { Serial number }\end{array}$ & $\begin{array}{l}\text { 物种 } \\
\text { Species }\end{array}$ & $\begin{array}{l}\text { 所属科 } \\
\text { Family }\end{array}$ & $\begin{array}{l}\text { 原始文献 } \\
\text { Original literature }\end{array}$ \\
\hline$\overline{1}$ & 灵山醉魂藤 Heterostemma tsoongii Tsiang & $\begin{array}{l}\text { 萝藦科 } \\
\text { Asclepiadaceae }\end{array}$ & $\begin{array}{l}\text { 中山大学农林植物研究所专刊 } \\
\text { Sunyatsenia 3: } 192.1936\end{array}$ \\
\hline 2 & 观光秋海棠 Begonia tsoongii C.Y. Wu & $\begin{array}{l}\text { 秋海棠科 } \\
\text { Begoniaceae }\end{array}$ & $\begin{array}{l}\text { 植物分类学报 } \\
\text { Acta Phytotax. Sin. 33(3): } 280 \text { (1995) }\end{array}$ \\
\hline 3 & $\begin{array}{l}\text { 阔片乌锁 Odontosoria tsoongii Ching } \\
\text { [=Odontosoria biflora (Kaulfuss) C. Christensen] }\end{array}$ & $\begin{array}{l}\text { 鳞始蒴科 } \\
\text { Lindsaeaceae }\end{array}$ & $\begin{array}{l}\text { 静生生物调查所汇报 } \\
\text { Bull. Fan Mem. Inst. Biol. 1: 149. } 1930\end{array}$ \\
\hline 4 & 观光鳞毛硕 Dryopteris tsoongii Ching & $\begin{array}{l}\text { 鳞毛蕨科 } \\
\text { Dryopteridaceae }\end{array}$ & $\begin{array}{l}\text { 中研院植物学汇报 } \\
\text { Bot. Res. Academia Sinica 2: } 14.1987\end{array}$ \\
\hline 5 & 假紫珠(钟君木) Tsoongia axillariflora Merr. & $\begin{array}{l}\text { 马鞭草科 } \\
\text { Verbenaceae }\end{array}$ & $\begin{array}{l}\text { 菲律宾科学杂志 } \\
\text { Philipp. J. Sci. 23: 264. } 1923\end{array}$ \\
\hline 6 & $\begin{array}{l}\text { 鸡嘴簕 Caesalpinia tsoongii Merr. [=Caesalpinia } \\
\text { sinensis (Hemsley) J. E. Vidal] }\end{array}$ & $\begin{array}{l}\text { 豆科 } \\
\text { Leguminosae }\end{array}$ & $\begin{array}{l}\text { 菲律宾科学杂志 } \\
\text { Philipp. J. Sci. 27: 162. } 1925\end{array}$ \\
\hline 7 & $\begin{array}{l}\text { 宽叶非(已合并) Allium tsoongii F. T. Wang \& } \\
\text { Tang [=Allium hookeri Thwaites] }\end{array}$ & $\begin{array}{l}\text { 百合科 } \\
\text { Liliaceae }\end{array}$ & $\begin{array}{l}\text { 静生生物调查所汇报 } \\
\text { Bull. Fan Mem. Inst. Biol. Bot. 7: 292. } 1937\end{array}$ \\
\hline 8 & $\begin{array}{l}\text { 观光木 Tsoongiodendron odorum Chun } \\
\text { [=Michelia odora (Chun) Noot. et B. L. Chen] }\end{array}$ & $\begin{array}{l}\text { 木兰科 } \\
\text { Magnoliaceae }\end{array}$ & $\begin{array}{l}\text { 植物分类学报 } \\
\text { Acta Phytotax. Sin. 8: 283. } 1963\end{array}$ \\
\hline 9 & 狭叶蒲桃 Eugenia tsoongii Merr. & $\begin{array}{l}\text { 桃金娘科 } \\
\text { Myrtaceae }\end{array}$ & $\begin{array}{l}\text { 菲律宾科学杂志 } \\
\text { Philipp. J. Sci. 21: 504. } 1922\end{array}$ \\
\hline 10 & 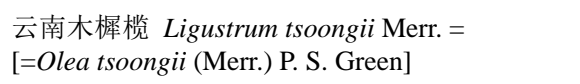 & $\begin{array}{l}\text { 木犀科 } \\
\text { Oleaceae }\end{array}$ & $\begin{array}{l}\text { 菲律宾科学杂志 } \\
\text { Philipp. J. Sci. 21: 506. } 1922\end{array}$ \\
\hline 11 & $\begin{array}{l}\text { 一掌参 Herminium tsoongii Tang \& F.T. Wang } \\
\text { [=Peristylus forceps Finet }]\end{array}$ & $\begin{array}{l}\text { 兰科 } \\
\text { Orchidaceae }\end{array}$ & $\begin{array}{l}\text { 北平研究院植物学研究所从刊 } \\
\text { Contr. Inst. Bot. Natl. Acad. } \\
\text { Peiping 2: 134. } 1934\end{array}$ \\
\hline 12 & $\begin{array}{l}\text { 钟氏柳 Salix tsoongii Cheng [=Salix dunnii var. } \\
\text { tsoongii (Cheng) C. Y. Yu et S. D. Zhao] }\end{array}$ & $\begin{array}{l}\text { 杨柳科 } \\
\text { Salicaceae }\end{array}$ & $\begin{array}{l}\text { 中国科学社生物研究所从刊 } \\
\text { Contrib. Biol. Lab. Sc. Soc. China, } \\
\text { Bot. Ser. x. } 68 \text { (1935) }\end{array}$ \\
\hline 13 & $\begin{array}{l}\text { 长柄赤车 Polychroa tsoongii Merr. } \\
\text { [=Pellionia latifolia (Blume) Boerl.] }\end{array}$ & $\begin{array}{l}\text { 寽麻科 } \\
\text { Urticaceae }\end{array}$ & $\begin{array}{l}\text { 菲律宾科学杂志 } \\
\text { Philipp. J. Sci. 21: 493. } 1922\end{array}$ \\
\hline 14 & $\begin{array}{l}\text { 长柄赤车 Pellionia tsoongii Merr. } \\
\text { [=Pellionia latifolia (Blume) Boerl.] }\end{array}$ & $\begin{array}{l}\text { 蕁麻科 } \\
\text { Urticaceae }\end{array}$ & $\begin{array}{l}\text { 岭南科学杂志 } \\
\text { Lingnan Sci. J. 6: } 325.1930 \text { [1928 publ. 1930] }\end{array}$ \\
\hline
\end{tabular}




\section{4 钟观光采集标本的意义}

钟观光的植物标本采集工作(1918-1921 年)是 首次由中国学者主持的大规模采集活动。他选择的 标本采集地有些是国外采集者的重点采集地如福 州、厦门、广州、昆明、大理、黄山等, 另一些如 防城港、韶关、苍梧等地是国外采集者较少涉及的 地方, 为我国学者了解中国植被, 尤其是南方植被 分布情况提供了第一手资料。钟观光采集的标本经 过研究发现包含新种 47 个(朱宗元和梁存柱, 2005), 著名蕨类学家秦仁昌研究了其中的偋类部分标本 并发表 7 个新种, 菊科专家林榕研究了菊科部分标 本, 禾本科专家耿以礼研究了禾本科部分, 方文培 研究了棫树科部分, 汪发瓒和唐进研究了兰科部分, 著名美国植物学家 E. D. Merril 研究了大部分钟观 光采集的标本并以一新属——钟君木属(Tsoongia) 来纪念他。随着研究的深入, 将会有更多的发现。 后来学者为了纪念钟观光先生的工作, 以他姓氏命 名的植物就有 14 种之多(表 3)。

钟观光的采集最大的特点是采集地域跨度大, 采集种类普遍，正如秦仁昌先生云: “北大标本之真 正价值, 不在于新种之多寡, 而在所经地域广大, 各类包罗宏富, 实为研究生态分布最完善之材料” (陈德禁和曾令波, 1988)。钟观光旅行采集日记在 《北京大学日刊》和《地学杂志》上的分期发表, 在 当时的生物学界产生了非常大的影响, 被尊为“中 国植物采集第一人”。其采集工作不仅开启了中国 当代分类学的新篇章, 也极大地鼓舞了中国学者对 中国本土的植物进行植物分类学研究。

植物分类学的目的之一是对植物物种进行系 统的分类和命名, 发现并命名新的植物种类是植物 学的基础。1840 年以后, 西方植物学开始传入中国 (刘学礼, 1991), 中国的现代植物学开始起步和发展, 然而当时植物学的教学都是利用欧美和日本的材 料, 如清华学校(清华大学前身)就购买了许多日本 的植物标本(陈桢, 1931)。真正的中国植物分类学研 究应该是以中国植物为材料, 确定其名称, 考察其 形态、性质和功用等(方文培, 1932)。北京大学以钟 观光采集的标本为基础建立植物标本室, 开辟了利 用我国植物材料进行植物学教学和研究之先河, 它 不仅是我国大陆最早的植物标本馆之一, 也为北京
大学生物系的创立奠定了基础。

致谢：感谢国家标本资源共享平台(NSII)、中国数字 植物标本馆 $(\mathrm{CVH})$ 、中国科学院植物研究所植物标 本馆(PE)、中国科学院昆明植物研究所标本馆(KUN) 以及美国哈佛大学植物标本馆 $(\mathrm{A} / \mathrm{GH}) 、$ 英国自然博 物馆植物标本馆 $(\mathrm{BM})$ 、爱丁堡植物园植物标本馆 (E)、英国皇家植物园邱园植物标本馆 $(\mathrm{K})$ 等提供的 资料。感谢中国科学院植物研究所覃海宁研究员、 浙江大学生命科学学院傅承新教授和赵云鹏教授、 福建师范大学生命科学学院陈炳华教授、湖南师范 大学刘克明教授的大力帮助。

\section{参考文献}

Bretschneider EV (1898) History of European Botanical Discoveries in China. Vol. I-II. Sampson Low, Marston and Company, London.

Chen DM, Zeng LB (1988) History evolution of China's botany-A brief history of China's botany systematics development. Journal of Central China Normal University (Nature Science), 22, 477-486. (in Chinese with English abstract) [陈德禁, 曾令波 (1988) 中国植物学发展史略一一植物 分类学发展简史. 华中师范大学学报(自然科学版), 22, 477-486.]

Chen JZ, Zhong RJ (2012) Tsoong Kuang-Kuan. In: A Survey of Academic Achievements of Chinese Famous Fcientists in the 20th Century, Biology (ed. Qian WC), pp. 3-12. Chinese Science and Technology of China Press, Beijing. (in Chinese) [陈锦正, 钟任建 (2012) 钟观光. 见: 20世纪中国知 名科学家学术成就概览(生物学卷, 钱伟长编), 3-12页. 中国科学技术出版社, 北京.]

Chen Z (1931) General situation about the department of biology in Tsinghua University. Tsinghua Weekly, 35(12), 75-80. (in Chinese) [陈桢 (1931) 国立清华大学生物系概 况. 清华周刊, 35(12), 75-80.]

Cox EM (1945) Plant-hunting in China: A History of Botanical Exploration in China and the Tibetan Marches, p. 89. Scientific Book Guild, London.

Fan WT, Chen YC (1990) Professor Tsoong Kuangkuan and our school botanical garden. Acta Agriculturae Universitatis Zhejiangensis, 16, 450-453. (in Chinese) [范文涛, 陈义产 (1990) 钟观光教授与我校植物园. 浙江农业大学学报, 16, 450-453.]

Fang WP (1932) Development history of botany in China. Scientific World, 1(2), 125-130. (in Chinese) [方文培 (1932) 中国植物学发展史略. 科学世界, 1(2), 125-130.]

Gao PS (1997) Chronicle of Cai Yuanpei (II). Peoples Education Press, Beijing. (in Chinese) [高平叔 (1997) 蔡元培年 谱长编(中). 人民教育出版社, 北京.]

Hao JS (1929) Department of biology in our university: Past 
and present. Peking University 31st Anniversary, 58-61. (in Chinese) [郝景盛 (1929) 我校生物学系的过去与现在. 北京大学卅一周年纪念刊, 58-61.]

Hao JS (1944) The botanical community in China during the past thirty years. Cultural Vanguard, 4(7), 5-8. (in Chinese) [郝景盛 (1944) 我国三十年来之植物学界. 文化先锋, 4(7), 5-8.]

Hu XS (1935) The progress of botany in China in the past twenty years. Science (China), 10, 1555-1559. (in Chinese) [胡先骕 (1935)二十年来中国植物学之进步. 科学, 10, 1555-1559.]

Jiang WQ (1941) Biography of Tsoong K. K. World Culture, 2(5), 57-61. (in Chinese) [蒋维乔 (1941) 钟观光传. 世界 文化, 2(5), 57-61.]

Jing Y (1940) In memory of Tsoong K. K. The Dah Loh Monthly, (6), 32-34. (in Chinese) [静渊 (1940) 记钟观光 先生. 大陆, 1(6), 32-34.]

Li HL (1944) Botanical exploration in China during the last twenty-five years. Proceedings of the Linnean Society of London, 156, 25-44.

Liu XL (1991) The introduction of western biology and the bud of modern biology in China. Journal of Dialectics of Nature, 76(6), 43-52. (in Chinese with English abstract) [刘学礼 (1991) 西方生物学的传入与中国近代生物学的萌芽. 自 然辨证法通讯, 76(6), 43-52.]

Ren RR (1980) In memory of Tsoong K. K. Plants, (6), 25-26. (in Chinese) [任荣荣 (1980) 怀念钟观光先生. 植物杂志, (6), 25-26.]

Sadahisa M (1911) A list of plants collected by Whang-i-jin in the Wai-shan, the Yu-shan, Mou-sek, Shong-Shuk and other places. Botanic Magazine, 25, 237-250. (in Japanese) [松田 定久 (1911) 黄以仁氏惠山虞山无锡常熟采集. 植物学 杂志, 25, 237-250.]

Wang FT (1949) Professor Kuan-kuang Tsoong. Contributions from the Institute of Botany, National Academy of Peiping, 6(1), 49-53.

Wang SZ, Ge MD, Chen SL (2005) Eighty years of wind and rain: A history review of School of Life Sciences (Department), Peking University. Life World, (4), 62-67. (in Chinese) [王世珍、葛明德、陈守良 (2005) 八十年风雨路北 京大学生命科学学院(系)历史回顾. 生命世界, (4), 62-67.]

Wang YZ (2006) A brief history of Chinese plant collection. In: Flora Reipublicae Popularis Sinicae, Tomus 1 (eds Wu CY, Chen SC), pp. 658-704. Science Press, Beijing. (in Chinese) [王印政 (2006) 中国植物采集简史. 见: 中国植 物志第一卷(吴征镒, 陈心启编), 658-704页. 科学出版
社, 北京.]

Wang ZC (1988) A concise history of modern biology of China. China Historical Materials of Science and Technology, 9(2), 17-37. (in Chinese with English abstract) [汪子春 (1988) 中国近代生物学发展概况, 中国科技史料, 9(2), 17-37.]

Wang ZX (1983) A brief history of botanical development in China. China Historical Materials of Science and Technology, (2), 22-30. (in Chinese) [王宗训 (1983) 中国植物学 发展史略. 中国科技史料, (2), 22-30.]

Wang ZX (1988) Review of modern Chinese botany. Plants, (4), 2-5. (in Chinese) [王宗训 (1988) 中国近代植物学回 顾. 植物杂志, (4), 2-5.]

Wang ZX (1993) History of Chinese Botany, pp. 121-143. Science Press, Beijing. (in Chinese) [王宗训 (1993) 中国 植物学史, 121-143页. 科学出版社, 北京.]

Xie ZS (1989) The Shanghai Scientific Instruments Company and the Word of Science. China Historical Materials of Science and Technology, 10(2), 61-66. (in Chinese with English abstract) [谢振声 (1989) 上海科学仪器馆与《科学世 界》.中国科技史料, 10(2), 61-66.]

Xie ZS (1990) A brief account of the Scientific Instruments Company. Science (China), 42(1), 70-71. (in Chinese) [谢 振声 (1990) 上海科学仪器馆述略. 科学, 42(1), 70-71.]

Yu YF, Chen JZ (1988) Tsoong Kuangkuan. in: The Current Biologists in China (ed. Tan JZ), pp. 1-11. Hunan Science \& Technology Press, Changsha. (in Chinese) [于一飞, 陈锦 正 (1988) 钟观光. 见: 中国现代生物学家(谈家祯编), pp. 1-11. 湖南科学技术出版社, 长沙.]

Zhang ZM (1947) Founder the Scientific Instruments Company. Yi Wen, 1, 23-24. (in Chinese) [张之铭 (1947) 创立 科学仪器馆之经过. 仪文, 1, 23-24.]

Zhu ZY, Liang CZ (2005) History of plant collection of professor Zhong Guanguang (Tsoong Kuan-Kuang) and establishment of the first herbarium in China. Acta Scicentiarum Naturalum Universitis Pekinensis, 41, 825-832. (in Chinese with English abstract) [朱宗元, 梁存柱 (2005) 钟观光先 生的植物采集工作：兼记我国第一个植物标本室的建立. 北京大学学报(自然科学版), 41, 825-832.]

Zou XD (2010) The History of College of Agriculture and Biotechnology Zhejiang University (1910-2010). Zhejiang University Press, Hangzhou. (in Chinese) [邹先定 (2010) 浙江大学农业与生物技术学院院史. 浙江大学出版社, 杭州.]

(责任编委：马克平 责任编辑: 黄祥忠)

\section{附录 Supplementary Material}

附录1 钟观光先生简历

Appendix 1 Introduction to Kuan-Kuang Tsoong http://www.biodiversity-science.net/fileup/PDF/2017328-1.pdf 
孟世勇, 刘慧圆, 余梦婷, 刘全儒, 马金双. 中国植物采集先行者钟观光的采集考证. 生物多样性, 2018, 26 (1): 79-88.

http://www.biodiversity-science.net/CN/10.17520/biods.2017328

\section{附录1＼cjkstart钟观光先生简历}

Appendix 1 Introduction to Kuan-Kuang Tsoong

钟观光 1869 年生于浙江镇海柴桥(钟观光, 钟氏宗谱); 1887 年考中秀才(陈锦正和钟任建, 2012); 1895 年甲午战争失败后, 有识之士痛感世变日亟, 非变革不足以自强, 钟观光深受维新思想的影响, 认识到恃 旧学不足以御侮, 而“科学为强国之根基”。他虽然无力深造, 却矢志自学理化等自然科学(蒋维乔, 1941); 1901 年 10 月 27 日在上海创立科学仪器馆, 向全国各中学或大学售卖科学仪器以及动植物标本(张之铭, 1947; 谢振生, 1990)。1901 年, 钟观光任江苏南菁高等学堂理化教员(陈梅龙和喻丹, 2007), 蒋维乔、黄以 仁是其当时的学生(蒋维乔, 1941; 谢振生, 2004)。1904 年, 科学仪器馆设立动植物标本制作所, 自行制作动 植物标本(谢振生, 1990); 同年, 钟观光因劳累过度到西湖养病, 期间自学李善兰等翻译的《植物学》, 从此 走上植物学研究的道路(蒋维乔, 1941; 任荣荣, 1980; 谢振生, 2004)。1909 年, 钟观光病愈后赴宁波旅沪公 学任博物教员(陈梅龙和喻丹, 2007)。1911 年, 受蔡元培之邀任教育部参事, 在放假期间与蔡元培、蒋维乔 步行采集植物于郊野(蒋维乔, 1941)。1915 年, 受蒋维乔推荐任湖南高等师范学校博物学教授, 在长沙周边 岳麓山等地采集植物标本(蒋维乔, 1941; 任荣荣, 1980)。1917 年起, 受聘北京大学地质系教授, 专门采集植 物标本。1918-1921 年, 在浙江、福建、广东、广西、云南、安徽、湖北等 11 个省采集植物标本 16,000 余 份。后于 1924 年参与创立了北京大学植物标本室 ${ }^{\mathbb{1}}$ (蒋维乔, 1941)。1927 年受聘浙江大学农学院教授, 任 标本部主任, 并到雁荡山、天目山等地采集植物标本 8,000 份; 1928 年建立杭州笕桥植物园。1933 年任职 北平研究院植物学研究所研究员, 进行植物名实考证研究。1936 年赴湖南考察森林植物。1 937 年因抗日战 争爆发返乡。1940 年于故乡去世。

\section{参考文献}

Chen JZ, Zhong RJ (2012) Tsoong Kuang-Kuan. In: A survey of Academic Achievements of Chinese Famous Fcientists in the 20th Century, Biology (ed. Qian WC), pp. 3-12. Science and Technology of China Press, Beijing. (in Chinese) [陈锦正, 钟任建 (2012) 钟观光. 见: 20世纪中国知名科学家学术成就概览(生物学卷, 钱伟长编), 3-12页. 中国科学技术出版社, 北京.]

Chen ML, Yu D (2007) The systematic botany founder in Chinese modern times - the brief biography of the famous educationalist Mr Cuangang Zhong. Journal of Ningbo Polytechnic, 11(6), 69-74. (in Chinese with English abstract) [陈梅龙, 喻丹 (2007) 中 国近代植物分类学的奠基人—著名教育家钟观光先生传略. 宁波职业技术学院学报, 11(6), 69-74.]

Jiang WQ (1941) Biography of Tsoong K. K. World Culture, 2(5), 57-61. (in Chinese) [蒋维乔 (1941) 钟观光传. 世界文化, 2(5), 57-61.]

Ren RR (1980) In memory of Tsoong K. K. Plants, 1980(6), 25-26. (in Chinese) [任荣荣 (1980) 怀念钟观光先生. 植物杂志, 1980(6), 25-26.]

Xie ZS (1990) A brief account of the Scientific Instruments Company . Science, 42(1), 70-71. (in Chinese) [谢振声 (1990) 上海科 学仪器馆述略. 科学, 42(1), 70-71.]

Xie ZS (2004) The pioneer of modern botany research in China -Tsoong Kuang-Kuan. Journal of the Party School of CPC Ningbo, 5, 93-95. (in Chinese) [谢振声 (2004) 我国近代植物学研究的先驱者一钟观光. 宁波党校学报, 5, 93-95.]

Zhang ZM (1947) Founder the Scientific Instruments Company. Yi Wen, 1: 23-24. (in Chinese) [张之铭 (1947) 创立科学仪器馆之 经过. 仪文, 1, 23-24.]

(1) 余梦婷 (2015) 钟观光的植物采集和植物学研究工作, 10-24 页. 硕士学位论文, 北京大学, 北京. 